$11,12,19$

\title{
Тепловые эффекты в окрестности температур фазовых переходов в матрично-изолированном нитрите натрия $\mathrm{NaNO}_{2}$
}

\author{
(C) В.М. Егоров ${ }^{1}$, Ю.Ф. Марков ${ }^{1, \uparrow}$, Е.М. Рогинский ${ }^{1}$, Е.В. Стукова ${ }^{2}$ \\ ${ }^{1}$ Физико-технический институт им. А.Ф. Иофрфе РАН, \\ Санкт-Петербург, Россия \\ ${ }^{2}$ Амурский государственный университет, \\ Благовещенск, Россия \\ 『 E-mail: yu.markov@mail.ioffe.ru \\ (Поступила в Редакцию 16 ноября 2016 г.)
}

\begin{abstract}
В ряде нанопористых силикатных матриц (с различными размерами пор), заполненных как из насыщенного раствора, так и из расплава, сегнетоэлектриком $\mathrm{NaNO}_{2}$, изучены тепловые эффекты в широкой температурной области, включая температуры фазовых переходов. Во всех образцах надежно обнаружены максимумы теплоемкости, соответствующие сегнетоэлектрическим фазовым переходам первого рода, получены характеристики этих максимумов (интенсивность, полуширина, температуры фазовых переходов и др.). Более сложная ситуация заключалась в обнаружении несоразмерной фазы - синусоидальной антисегнетоэлектрической, особенно, в случае размеров пор сравнимых с периодом „несоразмерной“ волны, проявление которой может быть объяснено возникновением соответствующей ориентации нанокристаллов нитрита натрия в порах этих матриц. Наблюдалась зависимость характеристик вышеуказанных эффектов от предистории изученных образцов.
\end{abstract}

DOI: 10.21883/FTT.2017.07.44600.415

\section{1. Введение}

В последние годы значительный интерес представляют исследования физических свойств систем с пониженной размерностью. Существуют различные способы получения наноматериалов. Одним из них является введение исследуемого вещества в нанопористую диэлектрическую матрицу. Характеристики этих композитных материалов определяются, главным образом, свойствами вводимых веществ, а также параметрами матрицы, в том числе, размерами пор и их геометрией. При наличии дипольных моментов у атомов введенного в матрицу вещества и малых межатомных расстояний, между ними возможна реализация диполь-дипольного взаимодействия, которое может также влиять на свойства этих наносистем. Влияние ограниченной геометрии изучалось на многих веществах, обладающих самыми различными свойствами. Надежно наблюдались размерные эффекты, в том числе, вблизи фазовых переходов. Индуцированные ограниченной геометрией изменения многих свойств, в основном, не противоречили теоретическим представлениям, развитым для изолированных малых частиц.

Наносегнетоэлектрики интересны как с научной точки зрения, так и с прикладной. Они имеют широкий спектр применения в электронике, как конденсаторы, как элементы энергонезависимой памяти, в качестве изоляторов, полевых транзисторов (FET), а также в других областях, в том числе, как нелинейные оптические устройства. Наноразмерные сегнетоэлектрики привлекают большое внимание из-за их уникальных физических свойств, которые отличаются от свойств объемных материалов. В настоящее время свойства сегнетоэлектриков, введенных в нанопористые матрицы, исследованы недостаточно, что, в значительной степени, обусловлено сложностью введения в нанопоры нерастворимых в воде сегнетоэлектрических материалов.

После открытия в кристаллах $\mathrm{NaNO}_{2}$ сегнетоэлектрического фазового перехода типа порядок-беспорядок $\left(T_{c}=436 \mathrm{~K}\right)$ это соединение стало модельным объектом широких исследований. Это связано с тем, что по сравнению с другими подробно изученными сегнетоэлектриками, демонстрирующими фазовые переходы того же типа (порядок-беспорядок), $\mathrm{NaNO}_{2}$ обладает простейшей кристаллической структурой. Уникальность фазового перехода нитрита натрия заключается в том, что простая сегнетоэлектрическая упорядоченная фаза отделена от неупорядоченной параэлектрической фазы синусоидальной антисегнетоэлектрической несоразмерной фазой, стабильной лишь в узком температурном интервале около $1-1.5 \mathrm{~K}$. Промежуточная фаза и детали ее синусоидальных полярных изменений содержат специфическую информацию об относительной важности учета близкодействующих и дипольных сил в $\mathrm{NaNO}_{2}$.

Результаты исследования нитрита натрия $\mathrm{NaNO}_{2}$, введенного в различные пористые матрицы (опалы, пористые стекла, молекулярные решетки), опубликованы в ряде работ (см., например, [1-5]). Среди множества полученных ранее результатов при изучении нано- $\mathrm{NaNO}_{2}$ можно отметить ряд наиболее ярких:

1. Обнаружение гигантского роста диэлектрической проницаемости в случае нанопористых матриц, заполненных $\mathrm{NaNO}_{2}$ в области температуры сегнетоэлектрического фазового перехода в объемных образцах [1]. 
2. Наблюдение в ряде работ (см., например, [2]) временно́й эволюции диэлектрических свойств к значениям, характерным для „объемного“ $\mathrm{NaNO}_{2}$.

3. При изучении дифракции нейтронов в условиях наноконфаймента $\mathrm{NaNO}_{2}$ в пористом стекле был обнаружен интересный эффект, а именно, аномальное возрастание амплитуды колебаний атомов натрия при температурах превышающих $T_{c}$ в объемных образцах $\mathrm{NaNO}_{2}$ [3].

4. В одной из работ (см. [4]) авторами предполагалось наличие состояния предплавления всего количества $\mathrm{NaNO}_{2}$, находящегося в порах, что, естественно, приводило к отсутствию сегнетоэлектрического фазового перехода.

5. Однако в другой работе [5] при изучении ЯМР аналогичных объектов привлекалась модель размытого плавления $\mathrm{NaNO}_{2}$, находящегося в порах, при котором часть $\mathrm{NaNO}_{2}$ была в расплавленном состоянии, а другая часть (с жесткой кристаллической решеткой) обладала свойствами, присущими объемному нитриту натрия.

Ранее уже изучались тепловые свойства (теплоемкость) нитрита натрия, введенного в наноразмерную пористую стеклянную матрицу с размерами пор $\sim 7 \mathrm{~nm}$. Наблюдался очень сильный сдвиг максимума теплоемкости в сторону низких температур и сильное размытие сегнетоэлектрического фазового перехода (размытый фазовый переход) и отсутствие скрытой теплоты перехода, но переход в несоразмерную фазу не был обнаружен [6].

Несколько позже были выполнены систематические калориметрические исследования также нитрита натрия, но введенного в наноматрицы с другим, большим размером пор - 23 и $160 \mathrm{~nm}$, когда наблюдались аномалии в поведении теплоемкости (гистерезис и др.), а в последнем случае $(160 \mathrm{~nm})$ было даже обнаружено проявление несоразмерной фазы [7].

Таким образом, очевидно, что в случае наноконфаймента этих объектов $\left(\mathrm{NaNO}_{2}\right)$ проявляются очень интересные и оригинальные явления, интерпретация которых не всегда однозначна, что в значительной степени может быть связано с недостатком экспериментального материала и недостаточной предварительной диагностикой изучаемых образцов. Все вышесказанное инициировало нас на новые исследования этих модельных и актуальных объектов.

\section{Методика эксперимента}

Исследовался ряд образцов нанопористых матриц

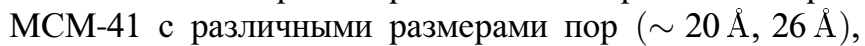
пористое стекло „Vycor“ (поры $\sim 70 \AA)$, а также технически важный природный цеолит (клиноптилолит), заполненных, как указывалось ранее, модельным сегнетоэлектриком $\mathrm{NaNO}_{2}$. Во всех случаях заполнение было выполнено из насыщенного раствора $\mathrm{NaNO}_{2}$ в дистиллированной воде, и лишь в случае использования в качестве матрицы пористого стекла „Vycor“ заполнение выполнялось из расплава $\mathrm{NaNO}_{2}$. Естественно, можно было предполагать, что исследуемые образцы будут несколько отличаться по свойствам, в том числе, по степени заполнения объема пор. В случае заполнения из расплава степень заполнения должна была быть выше ( 50\% объема пористой матрицы), чем в образцах, заполненных из раствора, что, по-видимому, и имело место. Для эталонирования экспериментальной техники и сравнения теплового поведения этих матричноизолированных образцов нами были выполнены калориметрические измерения объемных (порошкообразных) образцов химически чистого $\mathrm{NaNO}_{2}$. Тепловые свойства всех этих образцов исследовались на калориметре DSC-2 „Perkin-Elmer“ в атмосфере азота при варьировании скоростей нагревания и охлаждения в диапазоне $5-0.3 \mathrm{~K} / \mathrm{min}$. Температурная шкала калибровалась по точкам плавления льда $(273.1 \mathrm{~K})$ и индия $(429.7 \mathrm{~K})$, а шкала теплового потока - по теплоемкости лейкосапфира. Измерения проводились в области температур $250-450 \mathrm{~K}$. В измерениях использовались небольшие образцы, весом около $\sim 50 \mathrm{mg}$.

\section{2. Экспериментальные результаты и их обсуждение}

На рис. 1 приведено поведение теплоемкости, при нагреве и охлаждении, объемного нитрита натрия в области температур сегнетоэлектрического $\left(T_{c}=436 \mathrm{~K}\right)$ и несоразмерного фазовых переходов, которое демонстрирует два максимума, первый низкотемпературный и интенсивный соответствует сегнетоэлектрическому фазовому переходу І-го рода, а второй высокотемпературный максимум - менее интенсивный и более „широкий“ связан с фазовым переходом II-го рода в несоразмерную фазу. Температурный интервал между этими максимумами около $1-1.5 \mathrm{~K}$. В поведении обоих фазовых переходов наблюдается температурный гистерезис, который значителен в случае переходов

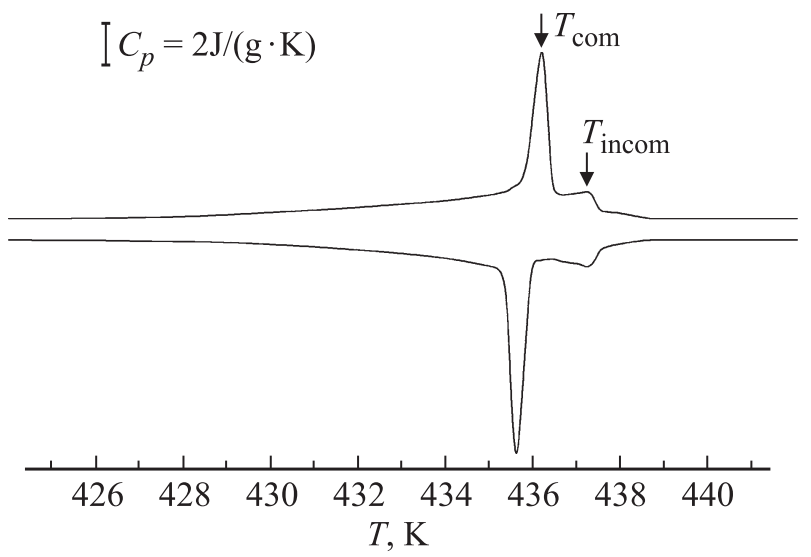

Рис. 1. Поведение теплоемкости в кристаллах $\mathrm{NaNO}_{2}$ (порошок) в области температур фазовых переходов (соразмерного и несоразмерного); верхняя кривая соответствует нагреву, нижняя — охлаждению. 


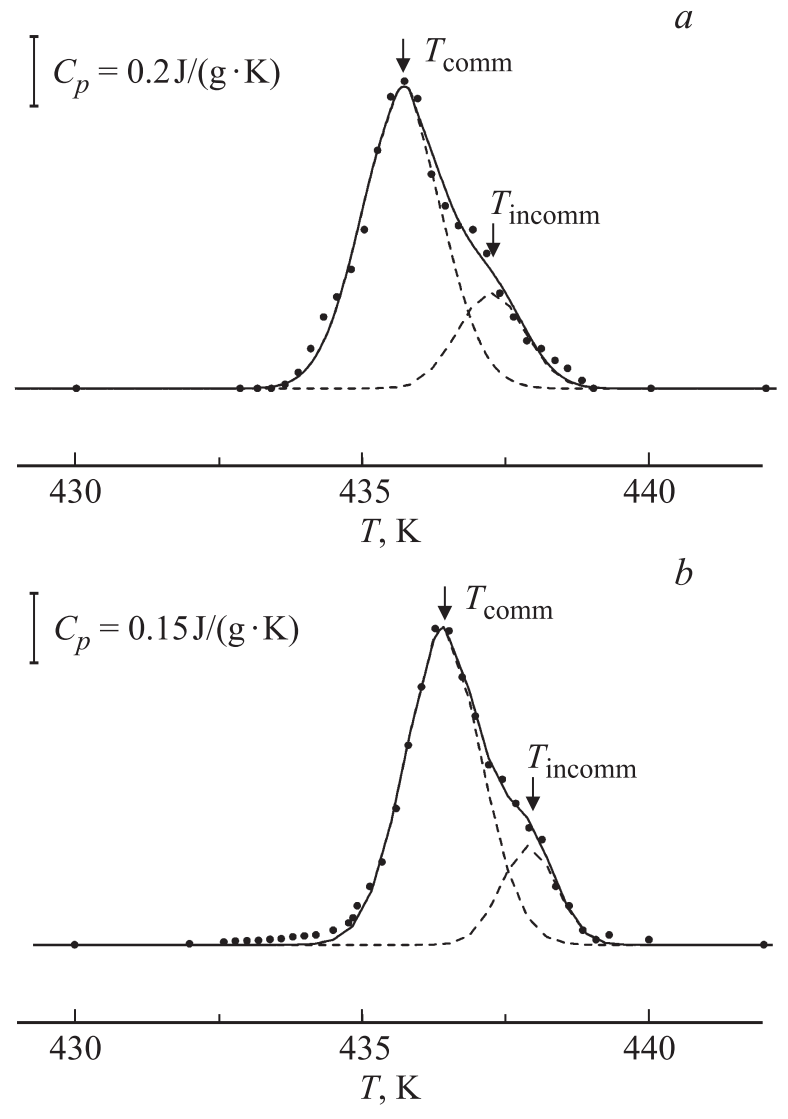

Рис. 2. Температурное поведение теплоемкости матричноизолированного $\mathrm{NaNO}_{2}$ в пористых матрицах. $(a)-\mathrm{NaNO}_{2}$ в матрице с размером пор $70 \AA,(b)$ - в матрице с размером пор $\sim 20 \AA$; здесь же выполнено разделение экспериментальных кривых на исходные контуры моделированием (точки эксперимент, сплошные линии - визуализация, штриховые линии - гауссианы).

I-го рода, что и можно было ожидать. Однако, и для несоразмерного фазового перехода имел место небольшой гистерезис, несколько противоречащий модели переходов II-го рода. Эти результаты, коррелирующие с известными литературными данными, см., например, [8], подтверждают достаточно высокий уровень состояния нашей экспериментальной техники и высокую чистоту нитрита натрия, использованного в работе, а также позволяют интерпретировать полученные результаты для случая матрично-изолированных наночастиц $\mathrm{NaNO}_{2}$ в сравнении с поведением чистого объемного $\mathrm{NaNO}_{2}$.

На рис. 2, $a, b$ приведено калориметрическое поведение двух нанообразцов $\mathrm{NaNO}_{2}$, сверху $(a)$ - температурное поведение теплоемкости $\mathrm{NaNO}_{2}$ в нанопористом стекле (размер пор $\sim 70 \AA$ ), а снизу $(b)-$ поведение теплоемкости матрично-изолированного $\mathrm{NaNO}_{2}$ (размер пор $\sim 20 \AA$ ); здесь же на рисунках выполнено разделение экспериментальных кривых на исходные контуры моделированием (точки - эксперимент, сплошные линии - визуализация, штриховые линии гауссианы).

Очевидно, что и в случае нанообразцов мы должны наблюдать интенсивные максимумы теплоемкости, соответствующие проявлениям структурного сегнетоэлектрического ФП І-го рода, что и имеет место. Как можно видеть, на этих двух рисунках в области температур сегнетоэлектрического фазового перехода проявляются уширенные максимумы, лишь немного (возможно, лишь в пределах погрешности) сдвинутые по температуре „вниз“ относительно $T_{c}$ в объемном $\mathrm{NaNO}_{2}$. Интегральные интенсивности этих максимумов - в объемном образце (порошок) и в матрично-изолированных сопоставимы с учетом степени заполнения изученных образцов.

Очевидно, что в случае этих 2-х нанообразцов $\mathrm{NaNO}_{2}$ (рис. 2, $a, b$ ) мы наблюдаем интенсивные максимумы теплоемкости, соответствующие проявлениям сегнетоэлектрического фазового перехода, что и должно быть. На высокотемпературных крыльях этих максимумов также надежно наблюдаются менее интенсивные пики, которые достаточно хорошо могут быть выделены математическим моделированием - разделением контуров на лоренцианы или гауссианы. Расстояния между этими максимумами около одного градуса $(1.2-1.5 \mathrm{~K})$, что полностью коррелирует с положениями соразмерного и несоразмерного фазовых переходов в объемных образцах $\mathrm{NaNO}_{2}$ (см. рис. 1). Положения всех этих максимумов теплоемкости нанообразцов $\mathrm{NaNO}_{2}$ немного сдвинуты в сторону низких температур относительно максимумов в „термограммах“ объемного $\mathrm{NaNO}_{2}$, что и можно было ожидать. Их полуширина значительно больше (в $\sim 3$ раза), чем в объемном $\mathrm{NaNO}_{2}$. Естественно, эти аномальные характеристики (главным образом, размытие) связать с проявлением сильной электрической и механической неоднородностей изученных нанообразцов $\mathrm{NaNO}_{2}$, в значительной степени индуцированной взаимодействием со стенками пор матриц. Общим свойством всех этих наносегнетоэлектрических кристаллов является размытие фазовых переходов при увеличении концентрации дефектов. Это размытие, хорошо проявляющееся на рис. 2, $a, b$ означает, что в сильно дефектных кристаллах сингулярность в поведении материальных констант исчезает. Можно предположить, что это связано с тем, что в таких кристаллах фазовый переход происходит не по всему объему образца при температуре $T_{c}$, а имеет место некоторое распределение локальных температур перехода, значения которых зависят от распределения неоднородностей, в том числе, дефектов по различным локальным областям нанокристаллов, точнее нанокристаллитов. Кроме того, при наличии полярных дефектов в сегнетоэлектрической фазе кристаллов может возникать внутреннее смещающее электрическое поле, также размывающее переход. Явления, связанные с этим полем, требуют отдельного и более строгого рассмотрения. 
Самое удивительное в „термограммах“ этих матрично-изолированных образцов $\mathrm{NaNO}_{2}$ - это проявление, и достаточно надежное, двух максимумов „соразмерного“ и „несоразмерного“. Проявление первого максимума, соответствующего сегнетоэлектрическому фазовому переходу первого рода и проявляющему обычно достаточно значительный, в несколько градусов, температурный гистерезис, вполне очевидно и не вызывает никаких вопросов. А вот второй, менее интенсивный максимум, убедительно проявляющийся в последних двух образцах (рис. 2, $a, b$ ), является более экзотичным, так как этот максимум, соответствующий несоразмерному фазовому переходу, ранее не удавалось наблюдать в „термограммах“, вплоть до размеров „объемных“ кристаллов. Необходимо учесть, что заполнение нанопор из насыщенных растворов $\mathrm{NaNO}_{2}$ или из расплава $\mathrm{NaNO}_{2}$, должно приводить к тому, что один-два монослоя, как минимум, имеют аморфную структуру, созвучную аморфному строению поверхности пор силикатных матриц (см., например, [9]). Таким образом, в нанопорах могут иметь место, как аморфная фаза, так и кристаллическая (даже монокристаллическая), последняя - на достаточном удалении от стенок пор, ближе к их центру. Несоразмерная фаза, синусоидально модулированная (с периодом $\sim 30-35 \AA$ ) по одному из кристаллографических направлений, требует определенной ориентации направления „замороженной волны“ - вдоль пор, т.е. вдоль максимальных размеров пор. Возможно, только в этом случае и можно ожидать проявления несоразмерной фазы в „термограммах“, особенно, при измерениях образцов с минимальными размерами пор $\sim$ единицы нанометров (в нашей работе).

На рис. 3, $a, b$ приведено более экзотичное температурное поведение теплоемкости нано- $\mathrm{NaNO}_{2}$ в других матрицах; сверху (a) на этом рисунке приведены „термограммы“ для случая $\mathrm{NaNO}_{2}$, введенного в модельный цеолит - природный клиноптилолит, имеющий три типа разноразмерных каналов, образующих двухмерную систему, с размерами входных окон от $\sim 4$ до $\sim 7.5 \AA$, представляющий также значительный прикладной интерес, а снизу (b) можно видеть калориметрические картинки нано- $\mathrm{NaNO}_{2}$, введенного в пористую матрицу с размерами пор $\sim 26 \AA$. Как видно из рисунка $3, a$ в случае матрицы клиноптилолита с $\mathrm{NaNO}_{2}$ наблюдается широкое и достаточно интенсивное низкотемпературное крыло, а также интенсивный максимум, соответствующий проявлению в „термограмме“ сегнетоэлектрического структурного фазового перехода. На высокотемпературном крыле, на расстоянии около $1.5 \mathrm{~K}$ от вышеуказанного максимума можно видеть размытый малоинтенсивный максимум, по-видимому, соответствующий несоразмерному фазовому переходу. Резкое уменьшение интенсивности этого максимума по сравнению с предыдущими образцами (см. рис. 1 и рис. $2, a, b$ ), инициировано значительным уменьшением размеров каналов и полостей в этом цеолите, ограничивающим вероятность

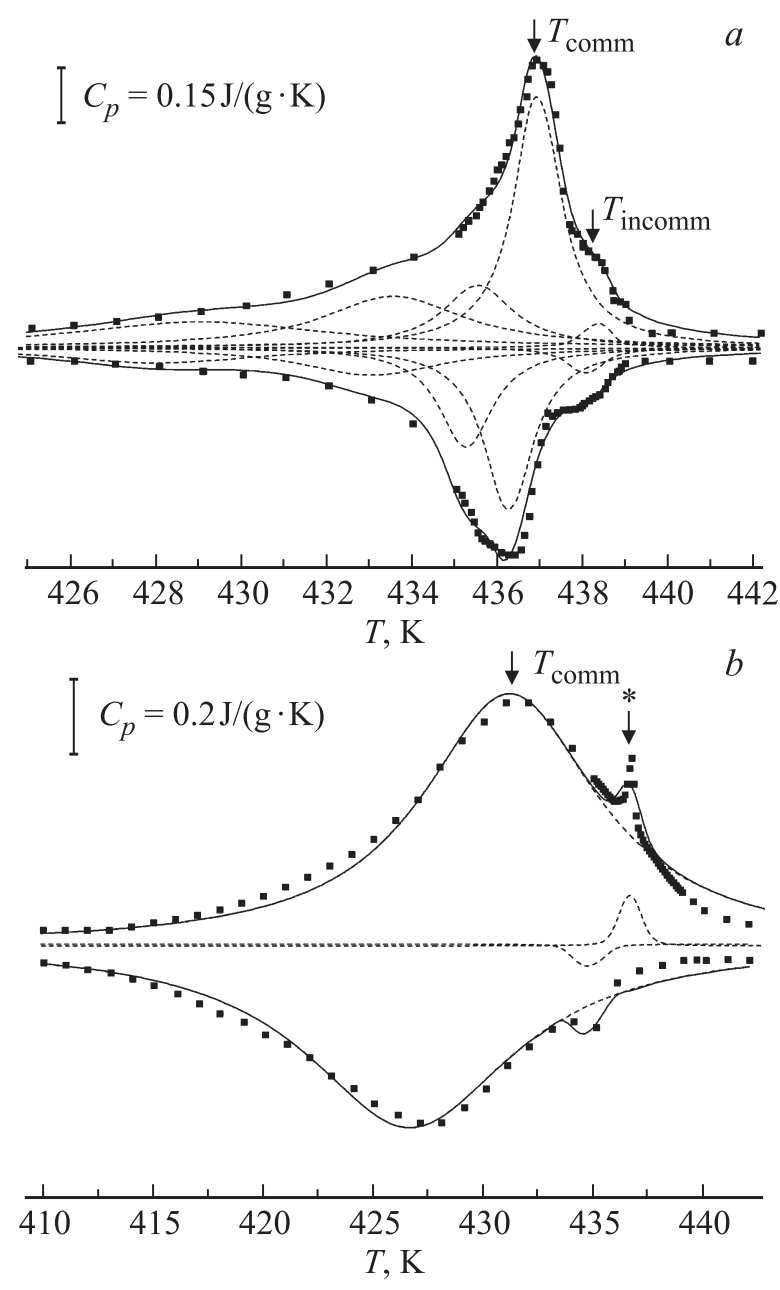

Рис. 3. Температурное поведение теплоемкости матричноизолированного $\mathrm{NaNO}_{2}$ в природном клиноптилолите $(a)$ и в матрице с размерами пор $\sim 26 \AA(b)$; верхние кривые соответствует нагреву, нижние - охлаждению (точки - эксперимент, штриховые линии - моделирование лоренцианами, сплошные линии - результат моделирования).

образования модулированной структуры и проявления несоразмерной фазы.

В принципе, можно попытаться объяснить всю эту аномальную картину (главным образом, низкотемпературное крыло) наличием большого количества заряженных и нейтральных дефектов матрицы, взаимодействующих с полярной фазой. Можно также выполнить разложение экспериментального контура, в том числе, и низкотемпературного крыла на ряд лоренцианов (гауссианов), что имеет под собой некоторые аргументы, так как в клиноптилолите имеется три типа двумерных каналов с различными размерами входных окон и каналов, что может приводить к трем локальным температурам структурных фазовых переходов $\mathrm{NaNO}_{2}$ в этой матрице. Величины гистерезиса всех этих локальных фазовых переходов при нагреве и охлаждении образцов не противоречат температурному гистерезису в чистом объемном $\mathrm{NaNO}_{2}$ (см. рис. 1). 
На рис. $3, b$ в случае нано- $\mathrm{NaNO}_{2}$, введенного в пористую матрицу с размерами пор $\sim 26 \AA$, наблюдается аномальная картинка относительно вышеуказанных, а именно, очень широкий максимум теплоемкости, сильно сдвинутый (на 5-6K) „вниз“ по температуре, что обычно имеет место в случае неравновесных, свежеприготовленных нанообразцов $\mathrm{NaNO}_{2}$. Похожие широкие максимумы теплоемкости нано- $\mathrm{NaNO}_{2}$ уже наблюдались в некоторых работах (см., например, [6,7]). На контуре этой диффузной полосы можно видеть также узкий пик (обозначен звездочкой), положение и полуширина которого, как при нагревании, так и при охлаждении, в том числе и гистерезис, коррелируют с соответствующими параметрами теплоемкости, связанными с соразмерным фазовым переходом, в объемных образцах нитрита натрия (см. рис. 1). Интенсивность этого пика соответствует наличию несвязанного в порах нитрита натрия, достигающего иногда несколько процентов от материала в порах. Проявление несоразмерного фазового перехода, по-видимому, скрыто в контуре широкого вышеуказанного максимума теплоемкости.

Здесь следует также отметить значительное влияние длительного хранения, т.е. старения образцов, на основные тепловые характеристики, в том числе, на параметры максимумов теплоемкости и на их положение (см. рис. 2, $a, b, 3 a, b)$. Значительное влияние на положение, полуширину и температурное поведение максимумов теплоемкости может оказывать также многократное прохождение изучаемых образцов через температуры фазовых переходов, главным образом, через температуру плавления (см., например, [2,5]). Обсуждаемые температурные опыты в значительной степени похожи на классическую термообработку твердых тел, плавление--отвердевание, отжиг-закалка, приводящие к перестройке и перекристаллизации изучаемых объектов, заполняющих нанопоры.

При выполнении экспериментов и интерпретации полученных результатов нельзя забывать здесь и о значительной гигроскопичности изучаемых сегнетоэлектриков $\mathrm{NaNO}_{2}$ при проведении различных прецизионных экспериментов, в том числе, диэлектрических, что может приводить к значительной погрешности экспериментальных результатов из-за присутствия свободной и связанной воды, в том числе, водных растворов $\mathrm{NaNO}_{2}$ различной концентрации. Следует заметить, что наличие нанопор значительно увеличивают эффекты адсорбции влаги в поры, и в конечном итоге, вода „поглощается“ изучаемым веществом. В экспериментах необходимо бороться с влиянием свободной и связанной воды и удалять ее с помощью предварительного прогрева, что является естественным и достаточно простым в калориметрических экспериментах

Итак, впервые в настоящей работе показано, что проявление фазовых переходов, в том числе несоразмерных, имеет место вплоть до минимальных размеров пор матрицы в $1-2 \mathrm{~nm}$. Можно утверждать, что соразмерные (из сегнетоэлектрической фазы в синусоидально модулированную, несоразмерную) и несоразмерные фазовые переходы (из антисегнетоэлектрической модулированной в парафазу) реализуются в этих материалах.

„Свежие“ образцы $\mathrm{NaNO}_{2}$ в нанопористых матрицах - обычно сильно неравновесны, и поэтому различные температурные манипуляции с ними, в том числе длительное хранение, приближают свойства этих образцов к свойствам объемных.

В заключение мы приносим благодарность П.Н. Якушеву за предоставление экспериментальной установки и возможности выполнить эти измерения.

\section{Список литературы}

[1] S.V. Pankova , V.V. Poborchii, V.G. Solovev. J. Phys.: Condens. Matter, 8, 12, L 203 (1996).

[2] Tien Cheng, E.B. Чарная, C.B. Барышников, M.K. Lee, S.Y. Sun, D. Michel, W. Böhlmann. ФTT 46, 12, 2224 (2004).

[3] A.V. Fokin, Yu.A. Kumzerov, N.M. Okuneva, A.A. Naberezhnov, S.B. Vakhrushev, I.V. Golosovsky, A.I. Kurbakov. Phys. Rev. Lett. 89, 17, 175503 (2002).

[4] S.B. Vakhrushev, Yu.A. Kumzerov, A. Fokin, A.A. Naberezhnov, B. Zalar, A. Lebar, R. Blinc. Phys. Rev. B 70, 13, 132102 (2004).

[5] С.В. Барышников, Е.В. Стукова, Е.В. Чарная, Tien Cheng, M.K. Lee, W. Böhlmann, D. Michel. ФTT 48, 3, 551 (2006).

[6] Z. Kutnjak, B. Vodopivec, R. Blinc, A.V. Fokin, Yu.A. Kumzerov, S.B. Vakhrushev. J. Chem. Phys. 23, 084708 ( 2005).

[7] E. Rysiakiewicz-Pasek, J. Komar, A. Cizman, R. Poprawski. J. Non-Crystalline Solids 356, 661 (2010).

[8] M. Sakiyama, A. Kimoto, S. Seki. J. Phys. Soc. Jpn., 20, 12, 2180 (1965)

[9] D. Wallacher, P. Huber, K. Knorr. J. Low Temperature Phys. 122, 3/4, 313 (2001). 